\section{Tension pneumoperitoneum following endoscopic submucosal dissection of leiomyoma of the cardia}

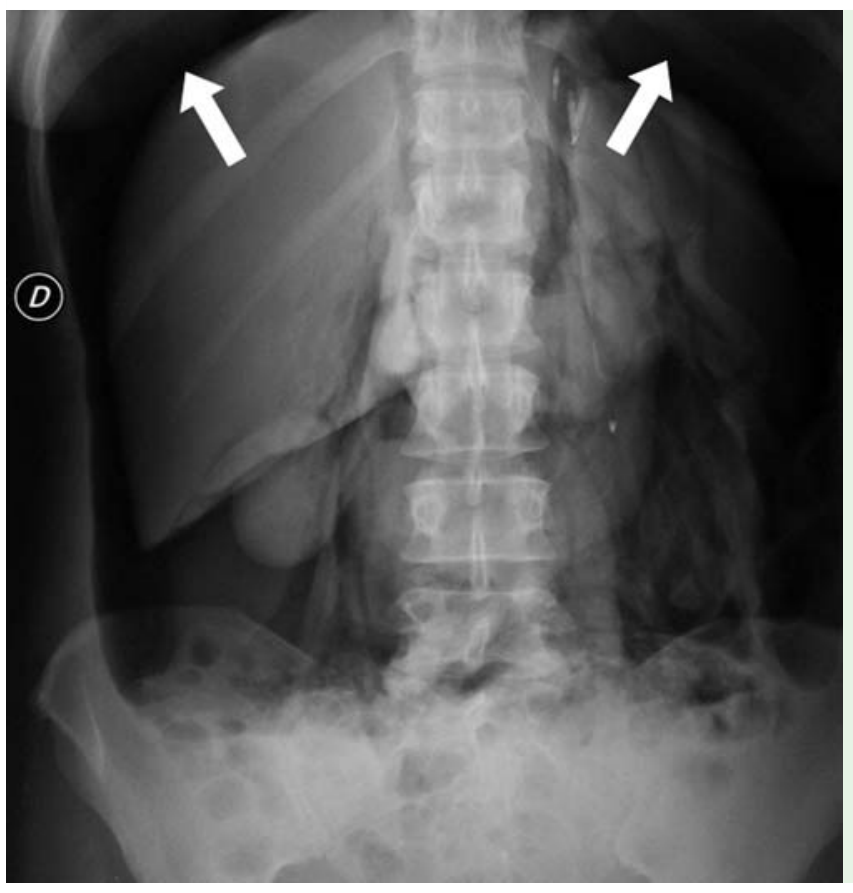

Fig. 1 Plain film of the abdomen taken two hours after the endoscopic procedure showing pneumoperitoneum (arrows).

A 39-year-old woman underwent video esophagography with barium because of persistent heartburn and regurgitation. A smooth defect with eccentric contours, about $2 \times 3 \mathrm{~cm}$ in size, was found at the esophagogastric junction. Upper gastrointestinal endoscopy confirmed the presence of a soft, polypoid, submucosal mass surrounding the cardia. Endoscopic ultrasonography showed a hypoechogenic, Cshaped lesion originating from the muscularis propria, with a fine echotexture consistent with leiomyoma. No biopsy was taken. The patient was considered a good candidate for endoscopic submucosal resection. The procedure was performed under general anesthesia with the patient in the supine position. A standard 9-mm endoscope with a soft transparent hood attached to its tip was advanced through an overtube into the stomach and then retroflexed. Enucleation was carried out after submucosal injection of $10 \mathrm{~mL}$ of diluted epinephrine using an insulated tip diathermic electrosurgical knife (IT-Knife 2; Olympus, Tokyo, Japan) at $100 \mathrm{~W}$ and a hook knife (Olympus Optical, Tokyo, Japan) at $60 \mathrm{~W}$. The dissection started along the lower border of the lesion and then extended circumferentially. Once the submucosal layer was reached, the tumor was gradually dissected away from the muscular layer and removed with an endoscopic bag. En bloc resection was achieved and the mucosal margins were sutured using three endoscopic clips. The procedure lasted 170 minutes. Histological examination confirmed the diagnosis of leiomyoma.

Postoperatively, the patient complained of severe, persistent abdominal pain unrelieved by analgesics and nasogastric intubation. Physical examination revealed generalized distension and tenderness of the abdomen, tachycardia, and mild hypotension. A plain film of the abdomen showed free air in the peritoneal cavity probably related to an air leak through the intact gastric wall ( $\bullet$ Fig. 1). Paracentesis was performed for decompression using a 20-gauge needle catheter. The patient recovered uneventfully after aspiration of about $3 \mathrm{~L}$ of air from the peritoneal cavity, and was discharged on postoperative day 3.

Endoscopic submucosal enucleation of tumors of the esophagogastric junction is a safe and effective technique in experienced hands. Overt perforation can occur, mainly on the gastric side, and can be managed by immediate clip application [1]. To our knowledge, this is the first reported case of tension pneumoperitoneum occurring after endoscopic submu- cosal dissection for leiomyoma at the esophagogastric junction. No definite perforation was recognized in our patient during the procedure, but severe symptoms developed in the postoperative period, requiring decompression paracentesis for relief. Interestingly, Fu et al. [2] described a similar complication during endoscopic submucosal dissection for adenoma of the cecum and the transverse colon. The procedure was discontinued, and an emergency abdominal computed tomography showed a large pneumoperitoneum with collapse of the inferior vena cava. The patient's symptoms were effectively relieved by paracentesis, and the blood pressure and diameter of the inferior vena cava returned to normal immediately after decompression.

In conclusion, tension pneumoperitoneum is a rare but potential complication of endoscopic submucosal dissection at the esophagogastric junction. Even in the absence of endoscopically detected perforation, prompt recognition and treatment of pneumoperitoneum is mandatory to prevent the cascade of events leading to cardiopulmonary complications and the abdominal compartment syndrome.

\section{Competing interests: None}

Endoscopy_UCTN_Code_CPL_1AH_2AZ

S. Siboni, D. Bona, E. Abate, L. Bonavina Department of Surgery, IRCCS Policlinico San Donato, University of Milan Medical School, Milan, Italy

\section{References}

1 Park Y, Park S, Kim T et al. Endoscopic enucleation of upper-GI submucosal tumors by using an insulated-tip electrosurgical knife. Gastrointest Endosc 2004; 59: 409-415

2 Fu K, Ishikawa T, Yamamoto T, Kaji Y. Paracentesis for successful treatment of tension pneumoperitoneum related to endoscopic submucosal dissection. Endoscopy 2009; 41: E245

\section{Bibliography}

DOI 10.1055/s-0029-1244164

Endoscopy 2010; 42: E152

(c) Georg Thieme Verlag KG Stuttgart · New York . ISSN 0013-726X

\section{Corresponding author \\ L. Bonavina \\ U.O. Chirurgia Generale \\ IRCCS Policlinico San Donato \\ Piazza Malan 2 \\ 20097 San Donato Milanese \\ Milan, Italy \\ Fax: +39-02-527746622 \\ luigi.bonavina@unimi.it}

